\title{
Direct observation of spin splitting in bismuth surface states
}

\author{
T. Hirahara,,${ }^{1} *$ K. Miyamoto, ${ }^{2}$ I. Matsuda, ${ }^{1}$ T. Kadono, ${ }^{2}$ A. Kimura, ${ }^{2}$ T. Nagao, ${ }^{3}$ G. Bihlmayer, ${ }^{4}$ E. V. Chulkov, ${ }^{5,6}$ S. Qiao, \\ K. Shimada, ${ }^{7}$ H. Namatame, ${ }^{7}$ M. Taniguchi,,${ }^{2,7}$ and S. Hasegawa ${ }^{1}$ \\ ${ }^{1}$ Department of Physics, University of Tokyo, 7-3-1 Hongo, Bunkyo-ku, Tokyo 113-0033, Japan \\ ${ }^{2}$ Graduate School of Science, Hiroshima University, 1-3-1 Kagamiyama, Higashi-Hiroshima 739-8526, Japan \\ ${ }^{3}$ Nano System Functionality Center, National Institute for Materials Science, 1-1 Namiki, Tsukuba, Ibaraki 305-0044, Japan \\ ${ }^{4}$ Institut für Festkörperforschung, Forschungszentrum Jülich, D-52425 Jülich, Germany \\ ${ }^{5}$ Donostia International Physics Center (DIPC), 20018 San Sebastián/Donostia, Spain \\ ${ }^{6}$ Departamento de Física de Materiales, UPV/EHU, Apartado 1072, 20080 San Sebastián, Spain \\ ${ }^{7}$ Hiroshima Synchrotron Radiation Center, Hiroshima University, 2-313 Kagamiyama, Higashi-Hiroshima 739-0046, Japan
}

(Received 6 April 2007; revised manuscript received 21 May 2007; published 12 October 2007)

\begin{abstract}
The electronic structure of ultrathin $\mathrm{Bi}(001)$ films on $\mathrm{Si}(111)-7 \times 7$ was studied by spin and angle-resolved photoemission spectroscopy. We directly observed a clear momentum-dependent spin splitting and polarization of the surface-state bands. The spin structure was antisymmetric with respect to the $\bar{\Gamma}$ point as predicted by theory, and the obtained in-plane spin polarization was as high as \pm 0.5 . The qualitative features of the observed spin polarization are discussed in comparison with the spin-polarized band structure obtained by first-principles calculations.
\end{abstract}

DOI: 10.1103/PhysRevB.76.153305

PACS number(s): 79.60.Bm, 68.35.-p, 73.20.-r, 85.75.-d

Spintronics, which aims at the utilization of the spin degree of freedom, has attracted wide interest due to its potential in realizing new functionalities in electronic devices. ${ }^{1}$ Spin manipulation is the key factor in spintronics, and the conventional style was to develop novel ferromagnetic materials. ${ }^{2}$ Recently, on the other hand, it was found that spin-split two-dimensional electron gases can be formed in asymmetric quantum wells controlled by an electric field even for nonmagnetic materials. ${ }^{3}$ This is called the Rashba effect, which is a combined effect of the spin-orbit interaction and structural inversion asymmetry (SIA). ${ }^{4}$

At the crystal surface, the same effect occurs and spinorbit split band structures have been found for $\mathrm{Au}(111)$ (Refs. 5 and 6) and W(110)-H (Ref. 7) surfaces. This splitting is caused by the spin-orbit coupling Hamiltonian, $H_{s o c}$ $=\left(\hbar / 4 m_{e}^{2} c^{2}\right) \vec{\sigma} \cdot(\nabla V \times \vec{p})$, where $\vec{\sigma}$ is the spin of electrons, $V$ the one-electron potential, and $\vec{p}$ the momentum. ${ }^{8}$ Usually in the bulk, the time-reversal symmetry $[E(\vec{k}, \uparrow)=E(-\vec{k}, \downarrow)]$ and the space-inversion symmetry $[E(\vec{k}, \uparrow)=E(-\vec{k}, \uparrow)]$ lead to the Kramers degeneracy. However, at the crystal surface, due to the SIA in the surface-normal direction, the degeneracy will be lifted. The spin orientation of such states is perpendicular both to the momentum $\vec{p}$ and to the electric field $\nabla V$, meaning an in-plane spin polarization antisymmetric about $\vec{k}=0$, as the electric field is perpendicular to the surface. $^{8}$

Bismuth (Bi) is a very heavy element and its electronic structure is highly influenced by the spin-orbit interaction. ${ }^{9}$ It was recently shown from angle-resolved photoemission spectroscopy (ARPES) measurements that the surface states of Bi crystals are highly metallic in contrast to the semimetallic nature of bulk Bi (Fig. 1). ${ }^{10-13}$ Additionally, it was suggested by $a b$ initio calculations that they will show large Rashba splitting due to the significant spin-orbit coupling. ${ }^{12,13}$ Furthermore, in a recent theoretical study, ${ }^{14}$ two-dimensional Bi bilayers were predicted to show the quantum spin Hall (QSH) effect, and it was said that these surface states may have some relations with the edge modes that characterize the QSH phase. The spin property of the highly metallic surface states of semimetallic Bi films also has importance in application to spintronics. However, although some insights about spin dependent scattering have been obtained by scanning tunneling spectroscopy, ${ }^{11,15,16}$ there has been no direct observation of the spin structure in a wide region of momentum-energy space. Spin and angleresolved photoemission spectroscopy (SRARPES) is a very powerful tool to determine the spin polarization and its $k$ dependence. Therefore, in the present study, we performed SRARPES measurements on ultrathin $\mathrm{Bi}(001)$ [hexagonal indexing, (111) in rhombohedral indexing] films and succeeded in resolving the spin-up and -down channels. Our results showed that the spin structure is antisymmetric with respect to the $\bar{\Gamma}$ point as predicted by theory, and the magnitude of in-plane spin polarization was as large as \pm 0.5 . Our $a b$ initio calculation reproduces the band topology of the films as well as the qualitative difference of the spin polar-
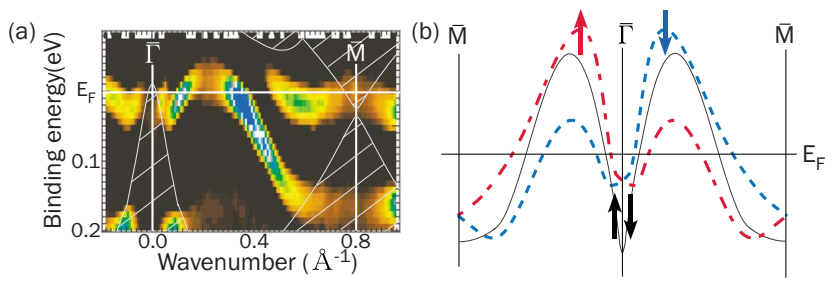

FIG. 1. (Color online) (a) The band structure of the surface state of a $17 \mathrm{BL}$ ultrathin $\mathrm{Bi}(001)$ film in the vicinity of $E_{F}$ along the $\bar{\Gamma}-\bar{M}$ direction measured by non-spin-resolved ARPES (Ref. 13). The shaded area represents the bulk band projection (Ref. 22). (b) Simulations of the dispersion of the surface states on the $\mathrm{Bi}(001)$ surface of a bulk crystal by first-principles calculations. The black solid lines are the results without considering spin-orbit coupling. The red short-dashed (blue broken) lines show the spin-up (-down) branch when the spin-orbit coupling is included (Ref. 12). 
ization for the spin-split states in the spin-orbit gap.

The measurements were performed in the SRARPES system at the Hiroshima Synchrotron Radiation Center. ${ }^{17,18}$ The spectra were recorded at room temperature using unpolarized He $I \alpha$ radiation $(21.2 \mathrm{eV})$ with a compact Mott detector operating at $25 \mathrm{keV} .{ }^{19}$ The angle between the incident photons and the analyzer was $50^{\circ}$. The angular resolution was $\pm 1^{\circ}$, and the energy resolution was $110 \mathrm{meV}$ for the pass energy $\left(E_{p}\right)$ of $5 \mathrm{eV}$ and $80 \mathrm{meV}$ for $E_{p}=3 \mathrm{eV}$. The data shown were recorded at $E_{p}=5 \mathrm{eV}$ unless otherwise indicated. In our measurement geometry, we can obtain the in-plane spin polarization of the spin component perpendicular to the plane spanned by the two vectors directed along the light incidence and electron emission, as shown in Fig. 2(a). We define the spin direction as "up" and "down" with respect to this plane, which can also be thought as "left" and "right" with respect to $k$. The spin polarization $P$ is obtained from $P=\left(1 / S_{e f f}\right)$ $\times\left[\left(I_{L}-I_{R}\right) /\left(I_{L}+I_{R}\right)\right]$, where $I_{L}$ and $I_{R}$ are the measured intensities of the left and right detectors, and $S_{\text {eff }}=0.128$ is the Sherman function. The spin-up and spin-down spectra are obtained by $I^{\uparrow, \downarrow}=\left(I_{L}+I_{R}\right)(1 \pm P) / 2$. Epitaxial $\mathrm{Bi}(001)$ films were grown in the method described before. ${ }^{13,20,21}$ The film thickness in the present measurement was $10 \mathrm{BL}$ (bilayers) ( $1 \mathrm{BL}=1.14 \times 10^{15}$ atoms $/ \mathrm{cm}^{2}, 0.39 \mathrm{~nm}$ thick).

First, we briefly discuss the physics of spin-orbit splitting of $\mathrm{Bi}(001)$ using the surface states at $E_{F}$ that have been extensively studied with ARPES. ${ }^{12,13}$ Figure 1(a) shows the band dispersion obtained by our previous study. ${ }^{13}$ The spin properties of these bands can be understood as follows. Figure 1(b) shows the band dispersion from the $a b$ initio calculations simulating the $\mathrm{Bi}(001)$ surface of a semi-infinite crystal without (black solid lines) and with (red short-dashed and blue broken lines) spin-orbit coupling included. ${ }^{12}$ Without the spin-orbit coupling, the spin-up and -down bands are degenerate that results in only two Fermi level crossings along $\bar{\Gamma}-\bar{M}$, while there are four bands crossing $E_{F}$ if they become split by the Rashba effect, which is consistent with the measured energy band dispersion of Fig. 1(a). However, whereas the two spin-split branches cross at $\bar{\Gamma}$ and $\bar{M}$ due to the combination of the time-reversal and the translational symmetry $[E(\vec{k}+\vec{G}, \uparrow)=E(\vec{k}, \uparrow)]$ in the semi-infinite case [Fig. 1(b)], we notice that the two bands do not cross at $\bar{M}$ for the Bi films [Fig. 1(a)]. In Ref. 13, we showed that the surface-state bands become quantum well states near $\bar{M}$ and the charge is no longer localized at the surface, weakening the spin splitting as well as modifying the band topology. The details concerning these points will be discussed below.

Now, let us turn to our SRARPES results. Figures 2(b) and 2(c) show the SRARPES spectra around normal emission. We find that spectral peaks are located at the binding energy $E_{B}=0.22-0.29 \mathrm{eV}$ for both spin channels, and their intensities are nearly the same although slight differences can be found at some emission angles (see the spectra at $\bar{\Gamma}$ ). As they are inside the bulk band projection ${ }^{13,20}$ [Fig. 4(b)] and the spin structure is not clearly antisymmetric with respect to $\bar{\Gamma}$, we conclude that these states are basically unpolarized. Relativistic selection rules in the photoexcitation process are presumably responsible for the small spin polarization observed. . $^{23,24}$
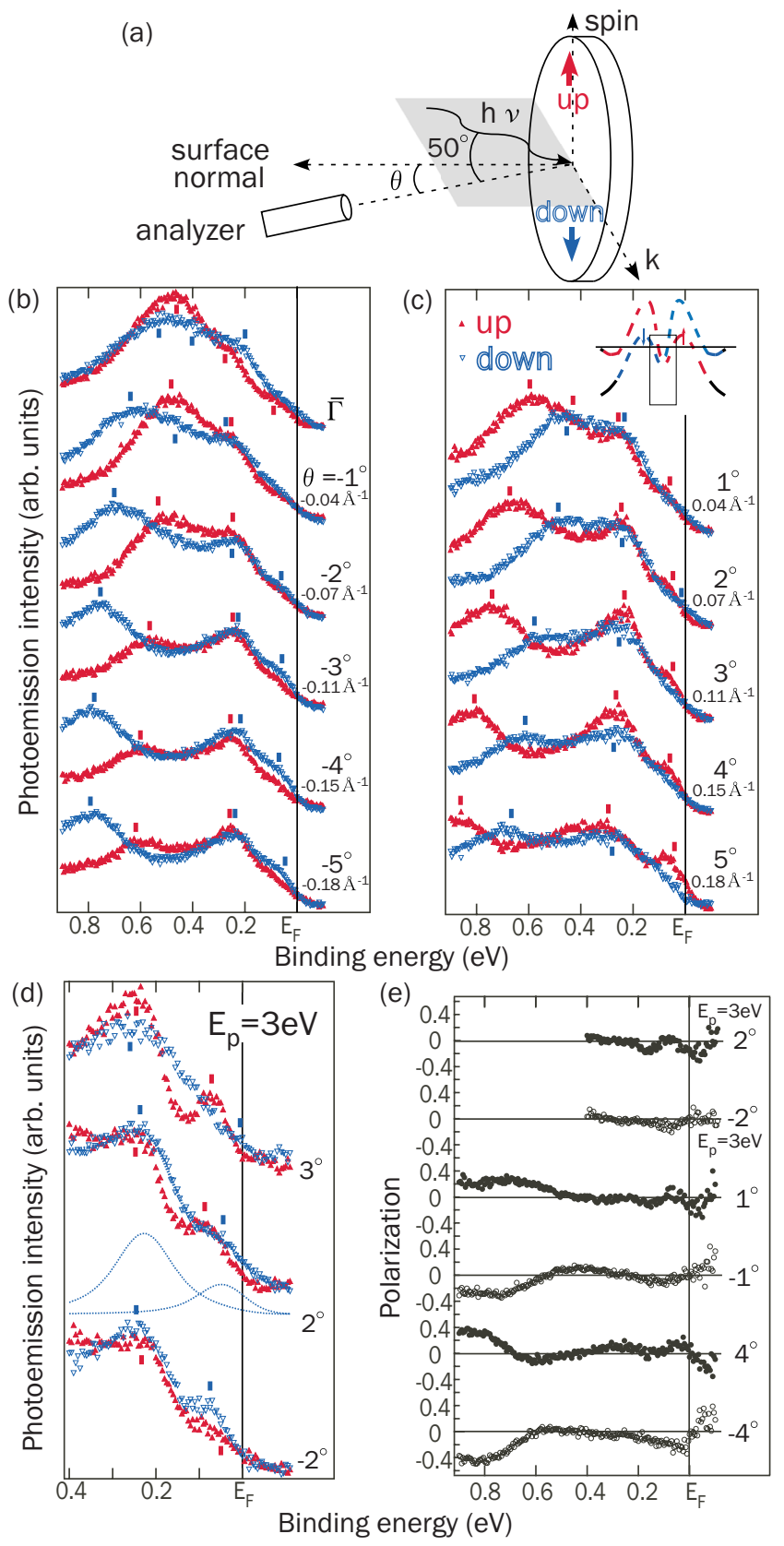

FIG. 2. (Color online) (a) The schematic drawing of the experimental setup. [(b) and (c)] The SRARPES spectra along the $\bar{\Gamma}-\bar{M}$ direction near $\bar{\Gamma}$ for the $10 \mathrm{BL} \mathrm{Bi(001)} \mathrm{film.} \mathrm{The} \mathrm{inset} \mathrm{in} \mathrm{(c)} \mathrm{shows}$ the measured region in the spin-split band structure [the red shortdashed and blue broken lines correspond to the spin-up and -down bands, respectively, and the black solid line shows the part where the polarization of the band is likely weakened as discussed in Fig. 4(b)]. (d) The SRARPES spectra taken with $E_{p}=3 \mathrm{eV}$ in the vicinity of $\bar{\Gamma}$. The dotted lines show the results of the peak fit described in the text. (e) The spin-polarization curves for some of the spectra shown in (b)-(d).

In contrast, there is a clear difference in the peak intensity between the spin-up and -down channels at $E_{B}$ $=0.5-0.9 \mathrm{eV}$. These states were identified as surface states inside the spin-orbit gap or resonances in the former 
(a)

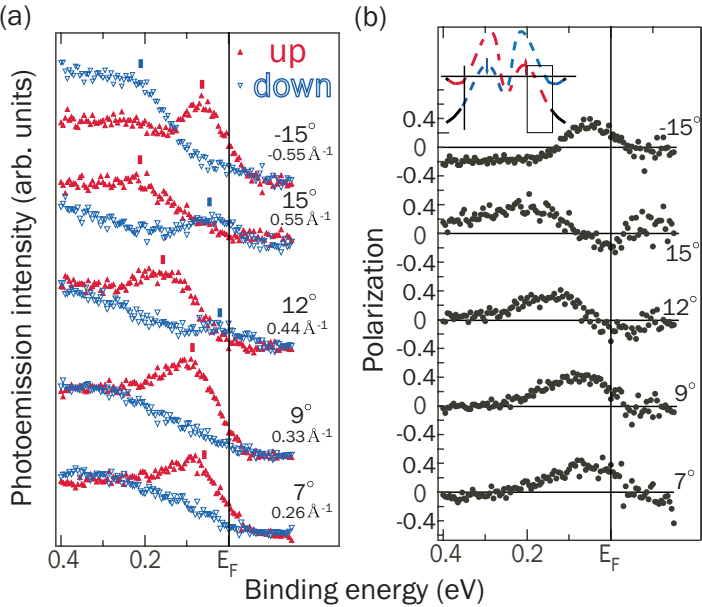

FIG. 3. (Color online) (a) The SRARPES spectra along the $\bar{\Gamma}-\bar{M}$

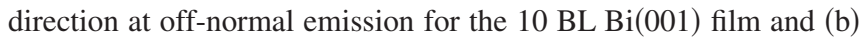
their corresponding spin polarization.

reports. ${ }^{13,20}$ The splitting behavior is evident, and the peak positions of the spins up and down are reversed between positive and negative emission angles, i.e., they are antisymmetric with respect to $\bar{\Gamma}$. In Fig. 2(e), the spin polarization for some of the spectra is shown. The in-plane spin polarizations are as high as \pm 0.5 for the states of higher $E_{B}$. We also notice that the peak width becomes somewhat broader and the spin polarization decreases a bit for these states as they approach $\bar{\Gamma}$. The spin-split states at lower $E_{B}$ show much smaller polarization than those at higher $E_{B}$. Furthermore, the splitting for these states at lower $E_{B}$ seems to vanish completely for the spectra at $\pm 1^{\circ}$, as peaks can be identified for both the spin-up and -down spectra at the same energy.

Just near $E_{F}$, we also found some difference between the spin-up and -down spectra. These states are the surface states described in Fig. 1. Their overall feature is consistent with the theoretical discussion as the spin-down states are observed at negative emission angles whereas the spin-up states for positive angles. However, we could not observe the spinsplit branch closer to $\bar{\Gamma}$ in Fig. 1(a) very clearly. Therefore, we have lowered $E_{p}$ and measured the spectra near $E_{F}$ again as shown in Fig. 2(d). The peaks become sharper in these spectra, and the state closer to $E_{F}$ is observed more clearly. The peak positions have been determined by fitting the spectra with Voigt functions with a Shirley-type background and a Fermi distribution function $(300 \mathrm{~K})$, as shown for the $2^{\circ}$ spin-down spectrum. The Gaussian width was fixed at $80 \mathrm{meV}$ (the experimental resolution) and the Lorentzian width was a fitting parameter, yielding $100-180 \mathrm{meV}$ for each peak. While further work is needed to determine the precise spin-polarization values, we believe that the splitting behavior is detected in these spectra.

Figures 3(a) and 3(b) show the SRARPES spectra at larger emission angles and the corresponding spin polarization, respectively. The spin-up band disperses downward for positive angles and a spin-down state also emerges at $12^{\circ}$. They correspond to the two spin-split branches shown in the inset in Fig. 3(b). We have also measured the spectra at $-15^{\circ}$, which show the reversed behavior as compared to those of

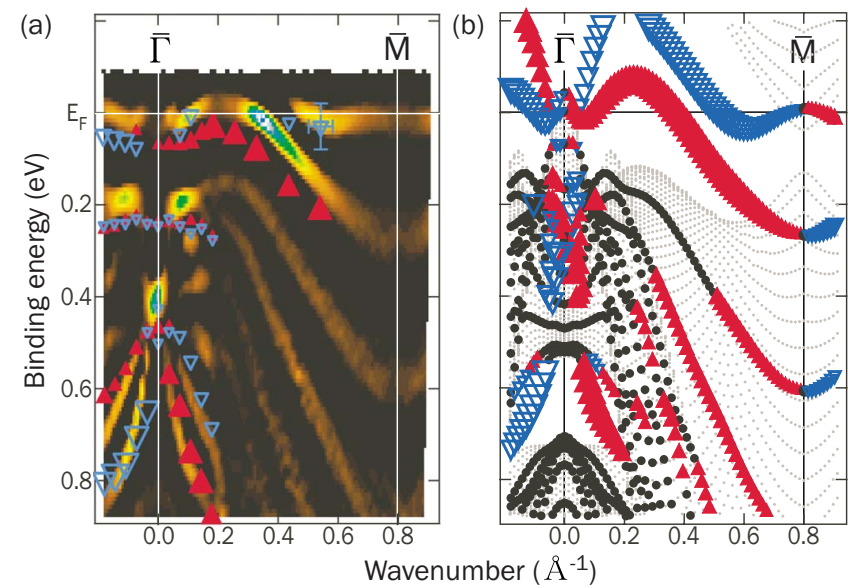

FIG. 4. (Color online) (a) The spin-split band dispersion of the $10 \mathrm{BL} \mathrm{Bi(001)} \mathrm{films} \mathrm{obtained} \mathrm{from} \mathrm{SRARPES} \mathrm{at} \mathrm{room} \mathrm{temperature}$ overlapped on the dispersion obtained by non-spin-resolved ARPES (Ref. 13) and (b) the spin-polarized band structure of the $a b$ initio calculations for freestanding Bi slabs. The spin-up (-down) channels are shown by the red solid triangle pointing up (blue empty triangles facing down), and the magnitude of the spin polarization is shown by the size of the markers. The states near $E_{F}$ in (a) have uncertainty in their positions indicated by the error bar owing to the resolution. The small gray dots in (b) represent the bulk band projection.

$15^{\circ}$. However, the intensity of the state closer to $E_{F}$ seems to be larger at $-15^{\circ}$ than at $15^{\circ}$. This may be due to the alignment of the photon incidence and electron emission, which will show asymmetric matrix element effects with respect to normal emission. ${ }^{25}$ The spin polarization of the peak at higher (lower) $E_{B}$ is $0.4-0.45$ ( -0.15 to -0.2$)$ for positive emission angles and is $-0.23(0.39)$ for the data at $-15^{\circ}$, respectively.

Figure 4(a) shows the spin-resolved energy band dispersion curves obtained from the present SRARPES measurement overlapped on the band dispersion image of the previous non-spin-resolved ARPES measurements taken at $\sim 130 \mathrm{~K} .{ }^{13}$ The size of the markers for the spin-polarized states represents the magnitude of the spin polarization. The largest markers represent \pm 0.5 and the smallest ones are $0 .{ }^{27}$ Unfortunately, we notice that the energy positions and dispersions slightly differ from our previous non-spin-resolved measurements. This is probably due to the poorer resolution in momentum and energy. Other reasons might be the thermally induced change in the dispersion ${ }^{26}$ which can be important for $\mathrm{Bi}$, or a slight misalignment of the scan direction as the dispersion of $\mathrm{Bi}$ surface states is anisotropic and steep. $^{20}$

Now, we compare our experimental results with the $a b$ initio calculations, ${ }^{28}$ shown in Fig. 4(b). The freestanding 10 BL film geometry is adopted for the calculation considering the weak interaction between $\mathrm{Bi}$ and $\mathrm{Si}$ as discussed in Ref. 13 , with the lattice constant taken as the experimental value in this case. In principle, the bands are spin degenerate or not spin polarized (black filled circles) due to the conservation of the space-inversion symmetry. However, the surface-state 
electron localized at one side of the slab is polarized in one direction, while the one at the other side has the opposite spin direction, and we have shown the polarization in the surface layer at one side in Fig. 4(b) (the largest triangles show \pm 0.97 ). The band dispersion obtained by ARPES in Figs. 1(a) and 4(a) (Ref. 13) is well reproduced along the whole $\bar{\Gamma}$ - $\bar{M}$ direction in Fig. 4(b). Also, as discussed above, the weakening of the spin polarization near $\bar{M}$ for the surface-state bands at $E_{F}$ is clearly seen. We also find in Fig. 4(b) the feature that the spin polarization is much smaller for the states at lower $E_{B}$ compared to those at higher $E_{B}$ in the spin-orbit gap near $\bar{\Gamma}$ at $0.5-0.9 \mathrm{eV}$, which was observed experimentally. The spin polarization obtained in the experiment is also influenced by matrix element effects, ${ }^{25}$ but the relative magnitude of the spin polarization for the surface states in Fig. 4(a) is qualitatively well reproduced by the calculation in Fig. 4(b).
For the states inside the bulk projection, no clear polarization was observed experimentally, whereas the calculation suggests some of them to be polarized. It would be interesting to perform measurements at lower temperature as well as relativistic one-step model SRARPES spectra calculations ${ }^{25}$ to identify the real polarization. ${ }^{29}$

In conclusion, we have shown the direct observation of a momentum-dependent spin splitting for the surface states of ultrathin $\mathrm{Bi}(001)$ films. As predicted from theory, the splitting was antisymmetric with respect to $\bar{\Gamma}$ and the in-plane spin polarization was as high as \pm 0.5 . Our first-principles calculation reproduces the band dispersion of the films as well as the qualitative difference of the spin polarization for the states of different branches in the spin-orbit gap.

This work has been supported by Grants-In-Aid from the Japanese Society for the Promotion of Science. *hirahara@surface.phys.s.u-tokyo.ac.jp

${ }^{1}$ S. A. Wolf, D. D. Awschalom, R. A. Buhrman, J. M. Daughton, S. von Molnar, M. L. Roukes, A. Y. Chtchelkanova, and D. M. Treger, Science 294, 1488 (2001).

${ }^{2}$ H. Ohno, Science 281, 951 (1998).

${ }^{3}$ T. Koga, J. Nitta, T. Akazaki, and H. Takayanagi, Phys. Rev. Lett. 89, 046801 (2002).

${ }^{4}$ E. I. Rashba, Sov. Phys. Solid State 2, 1109 (1960).

${ }^{5}$ S. LaShell, B. A. McDougall, and E. Jensen, Phys. Rev. Lett. 77, 3419 (1996).

${ }^{6}$ M. Hoesch, M. Muntwiler, V. N. Petrov, M. Hengsberger, L. Patthey, M. Shi, M. Falub, T. Greber, and J. Osterwalder, Phys. Rev. B 69, 241401(R) (2004).

${ }^{7}$ M. Hochstrasser, J. G. Tobin, E. Rotenberg, and S. D. Kevan, Phys. Rev. Lett. 89, 216802 (2002).

${ }^{8} \mathrm{R}$. Winkler, Spin-Orbit Coupling Effects in Two-Dimensional Electron and Hole Systems (Springer-Verlag, Berlin, 2003).

${ }^{9}$ X. Gonze, J.-P. Michenaud, and J.-P. Vigneron, Phys. Rev. B 41, 11827 (1990).

${ }^{10}$ C. R. Ast and H. Höchst, Phys. Rev. Lett. 87, 177602 (2001).

${ }^{11} \mathrm{Ph}$. Hofmann, Prog. Surf. Sci. 81, 191 (2006).

${ }^{12}$ Y. M. Koroteev, G. Bihlmayer, J. E. Gayone, E. V. Chulkov, S. Blügel, P. M. Echenique, and Ph. Hofmann, Phys. Rev. Lett. 93, 046403 (2004).

${ }^{13}$ T. Hirahara, T. Nagao, I. Matsuda, G. Bihlmayer, E. V. Chulkov, Yu. M. Koroteev, P. M. Echenique, M. Saito, and S. Hasegawa, Phys. Rev. Lett. 97, 146803 (2006).

${ }^{14}$ S. Murakami, Phys. Rev. Lett. 97, 236805 (2006).

${ }^{15}$ J. I. Pascual, G. Bihlmayer, Y. M. Koroteev, H.-P. Rust, G. Ceballos, M. Hansmann, K. Horn, E. V. Chulkov, S. Blügel, P. M. Echenique, and Ph. Hofmann, Phys. Rev. Lett. 93, 196802 (2004).

${ }^{16}$ T. K. Kim, J. Wells, C. Kirkegaard, Z. Li, S. V. Hoffmann, J. E. Gayone, I. Fernandez-Torrente, P. Haberle, J. I. Pascual, K. T. Moore, A. J. Schwartz, H. He, J. C. H. Spence, K. H. Downing, S. Lazar, F. D. Tichelaar, S. V. Borisenko, M. Knupfer, and Ph.
Hofmann, Phys. Rev. B 72, 085440 (2005).

${ }^{17}$ K. Iori, K. Miyamoto, H. Narita, K. Sakamoto, S. Qiao, A. Kimura, K. Shimada, H. Namatame, and M. Taniguchi, J. Electron Spectrosc. Relat. Phenom. 144-147, 997 (2005).

${ }^{18}$ K. Iori, K. Miyamoto, H. Narita, K. Sakamoto, A. Kimura, S. Qiao, K. Shimada, H. Namatame, and M. Taniguchi, Rev. Sci. Instrum. 77, 013101 (2006).

${ }^{19}$ S. Qiao, A. Kimura, A. Harasawa, M. Sawada, J.-G. Chung, and A. Kakizaki, Rev. Sci. Instrum. 68, 4390 (1997).

${ }^{20}$ T. Hirahara, T. Nagao, I. Matsuda, G. Bihlmayer, E. V. Chulkov, Yu. M. Koroteev, and S. Hasegawa, Phys. Rev. B 75, 035422 (2007).

${ }^{21}$ T. Nagao, J. T. Sadowski, M. Saito, S. Yaginuma, Y. Fujikawa, T. Kogure, T. Ohno, Y. Hasegawa, S. Hasegawa, and T. Sakurai, Phys. Rev. Lett. 93, 105501 (2004).

${ }^{22}$ Y. Liu and R. E. Allen, Phys. Rev. B 52, 1566 (1995).

${ }^{23}$ C. M. Schneider, J. Garbe, K. Bethke, and J. Kirschner, Phys. Rev. B 39, 1031 (1989).

${ }^{24}$ K. Miyamoto, K. Sakamoto, H. Narita, A. Kimura, M. Taniguchi, S. Qiao, K. Hasegawa, K. Shimada, and H. Namatame, (unpublished).

${ }^{25}$ J. Henk, M. Hoesch, J. Osterwalder, A. Ernst, and P. Bruno, J. Phys.: Condens. Matter 16, 7581 (2004).

${ }^{26}$ J. A. Knapp, F. J. Himpsel, A. R. Williams, and D. E. Eastman, Phys. Rev. B 19, 2844 (1979).

${ }^{27}$ The states that show clear splitting but do not clearly show up in the spin-polarization curve are also shown as 0, e.g., the peak close to $E_{F}$ of the $-2^{\circ}$ spin-up spectrum.

${ }^{28}$ The calculations have been performed using the full-potential linearized augmented plane wave method in film geometry as implemented in the FLEUR program and local density approximation for the description of exchange correlation potential.

${ }^{29}$ We have actually tried to measure the states in the bulk band projection near $\bar{M}$, but the intensity becomes drastically weak and the quality of the spectra is not good enough to assign peak positions. 